\title{
REPRESENTACIÓN DE LOS NÚMEROS REALES EN LA RECTA
}

\author{
CORIAT, MOISÉS y SCAGLIA, SARA ${ }^{1}$ \\ Grupo de Pensamiento Numérico. Departamento de Didáctica de la Matemática. Universidad de Granada. \\ ${ }^{1}$ Profesora de la Universidad Nacional del Litoral. Santa Fe. Argentina. \\ Becaria FOMEC en la Universidad de Granada.
}

\begin{abstract}
SUMMARY
We state and justify a conjecture concerning the comparative complexity of the representation of real numbers on the line versus other representations. The Introduction provides the setting of the subject and states the conjecture. Part 1 deals with the controversial line's «nature» and depicts several interpretations of the straight line. Part 2 analyses the particular phenomenology related to the assignment of real numbers to the line's points, in order to extract some limitations of such an assignment. Part 3 compares several representations of real numbers with the representation on the line, and presents a set of distinctive features for the latter.

This paper, theoretically oriented, comes from a broader research work addressed to uncover epistemological obstacles related to the representation of real numbers on the straight line. We are doing a work field, involving students of several levels, on this subject.
\end{abstract}

\section{INTRODUCCIÓN}

Desde el punto de vista escolar, la «recta» viene a ser como un soporte de números que progresivamente se va «completando». En primaria se comienza «poniendo» naturales y en bachillerato se acaba situando reales, que ya no dejarían «huecos» en ella: fijados dos puntos distintos, que representan respectivamente el cero y la unidad, se establece una aplicación biyectiva entre el conjunto de números reales y el conjunto de puntos de la recta. Muchos libros sancionan esta práctica habitual en el medio educativo; por ejemplo, un conocido diccionario de matemáticas (Bouvier et al., 1984) define recta numérica como «conjunto ordenado de los números reales $\mathrm{R}$ ». Esta definición y otras análogas se apoyan en la amplia difusión, coherencia y aplicación de la hoy clásica interpretación debida a Cantor y Dedekind.

Aunque esta descripción, necesariamente escueta, parezca esencialmente correcta, omite algunos problemas matemáticos, epistemológicos y educativos.
En primer lugar, la esencial aportación de Cantor y Dedekind ha permitido que la discusión sobre la «naturaleza de la recta», prosiga, dentro de la matemática, a lo largo del presente siglo, a través de diferentes axiomáticas.

En segundo lugar, aunque esta discusión se realice a través de argumentos formales, pone en juego intuiciones esencialmente diferentes; ahora bien, las intuiciones son duraderas y se expresan ya desde la edad escolar. Fischbein (1987, p. 211) sostiene que «el problema educativo no es eliminar las representaciones e interpretaciones intuitivas, sino desarrollar la capacidad del estudiante para analizar y poner bajo control sus concepciones intuitivas y construir nuevas intuiciones consistentes en los requerimientos científicos normales». De hecho, tampoco hay coincidencia acerca de las intuiciones que se puedan tener de la recta como objeto mental (Solomon, 1991). Las intuiciones de la recta son varia- 
das y discrepantes, incluso contradictorias, como algunas investigaciones ponen de manifiesto (Mansfield, 1985; Romero, 1996).

En tercer lugar, aunque la biyección punto-número, aparentemente, describa un simple emparejamiento de todos y cada uno de los puntos de la recta con todos y cada uno de los números reales, aún tenemos que resolver la cuestión de la atribución de etiquetas numéricas a los puntos, y esto constituye un problema que no es exclusivamente procedimental, sino también conceptual, como se pondrá de manifiesto. En bachillerato se podrían evitar discusiones epistemológicas «decretando» que la recta geométrica «se llena» con los números reales, pero la complejidad del concepto de número real no es evitable ${ }^{1}$. Al referirse a la complejidad de $R$, Romero (1995) propone que se combinen los sistemas de representación «notación decimal» y «modelo de la recta». Si adoptamos la terminología de Romero, parecería que el sentido del propio «modelo de la recta» obliga a incorporar otros sistemas de representación. Dicho como conjetura: la representación de números reales en la recta es más compleja que otras representaciones de estos números, al menos en la educación secundaria.

En este trabajo tratamos brevemente tres asuntos. La parte titulada «Cuestiones epistemológicas; datos para un análisis conceptual» aborda selectivamente la controversia en torno a la «naturaleza» de la recta geométrica y recoge algunas interpretaciones diferentes de la recta de origen matemático. Junto a una interpretación hoy estándar, iniciada por Cantor y Dedekind, describiremos algunas características de otras interpretaciones debidas a Robinson y a Veronese. Las concepciones de estos últimos autores, si bien no coinciden, tienen en común la adición de objetos infinitesimales (puntos o segmentos, respectivamente) a la recta. La parte titulada «Cuestiones fenomenológicas; un análisis conceptual» analiza la asignación concreta de números reales a puntos de la recta. Ponemos de manifiesto limitaciones de la propia asignación punto-número y la necesidad de ampliar la noción de número constructible si se quiere dar más seguridad a una creencia, básica en secundaria, según la cual la biyección punto-número se podría realizar efectivamente para todo número real. La última parte, «Representación en la recta y otras representaciones», compara diferentes representaciones de números reales con la representación en la recta y termina con una colección de características distintivas de esta última, dando así -esperamos- crédito y sentido a la conjetura que hemos enunciado en esta misma introducción.

Debemos hacer también dos breves apuntes de carácter metodológico:

a) Cuando hablamos de representaciones, nos referimos exclusivamente a representaciones externas. Más precisamente, admitimos que hay conceptos (como recta geométrica, número real) y representaciones públicamente compartidos. Cualquier referencia a representaciones internas, en este trabajo, proviene, exclusivamente, de citas ajenas (como la ya usada de Solomon). La anterior afirmación no implica un rechazo de las representaciones internas; más bien, entendemos que es una exigencia del análisis conceptual.

b) El análisis conceptual (Scriven, 1988) constituye una herramienta de indagación filosófica reconocida como metodología de investigación. Lo hemos realizado, partiendo de producciones ajenas, usando sistemáticamente el siguiente guión: uno de nosotros sugería análisis concretos que el otro supervisaba, comentaba o criticaba, produciéndose así un feedback destinado, entre otras cosas, a asegurar que las representaciones usadas son compartidas (externas).

\section{CUESTIONES EPISTEMOLÓGICAS. DA- TOS PARA UN ANÁLISIS CONCEPTUAL}

La controversia en torno a la «naturaleza» de la recta geométrica es casi tan antigua como la filosofía. No es nuestro propósito contar su historia, sino recoger una selección de aportaciones originadas en el seno de las propias matemáticas y que han tenido lugar a lo largo del siglo $\mathrm{xx}^{2}$. Esta parte presenta algunas cuestiones que atañen a la aceptación de una correspondencia uno a uno entre los números reales y los puntos de la recta. La aceptación de esta correspondencia supone «tomar partido» acerca de la estructura de la recta.

Según Crossley (1987, p. 152), «fue Cantor quien primero señaló explícitamente que la identificación del sistema de números con puntos sobre la recta era una asunción que no podría ser demostrada, aunque parecía plausible y psicológicamente convincente -y aún lo parece a muchos matemáticos de hoy». El axioma que introduce Cantor para garantizar la correspondencia entre los números reales y los puntos de la recta afirma que, fijados un punto como origen y una unidad de medida, cada cantidad numérica tiene un punto determinado sobre la línea recta, cuya coordenada es igual a esta cantidad (Belna, 1996, p. 134).

También Dedekind (1998, pp. 84-85) reconoce que la continuidad de la recta es necesario expresarla mediante algún axioma: «Si todos los puntos de la recta se descomponen en dos clases tales que todo punto de la primera clase está a la izquierda de cada punto de la segunda clase, entonces existe uno y sólo un punto que produce esta partición de todos los puntos en dos clases, este corte de la recta en dos partes.»

Dedekind, después de expresar su deseo de que todos encuentren su axioma evidente y coincidente con su representación de recta, indica la imposibilidad de manifestarse acerca de la verdadera naturaleza de la recta: «La suposición de esta propiedad de la línea no es más que un axioma mediante el cual atribuimos a la línea por vez primera su continuidad, mediante el cual introducimos la continuidad en nuestra idea de línea. Si el espacio tiene una existencia real, sin duda no es necesario que sea continuo; innumerables propiedades suyas seguirían siendo las mismas aunque fuera discontinuo. Y si supié- 


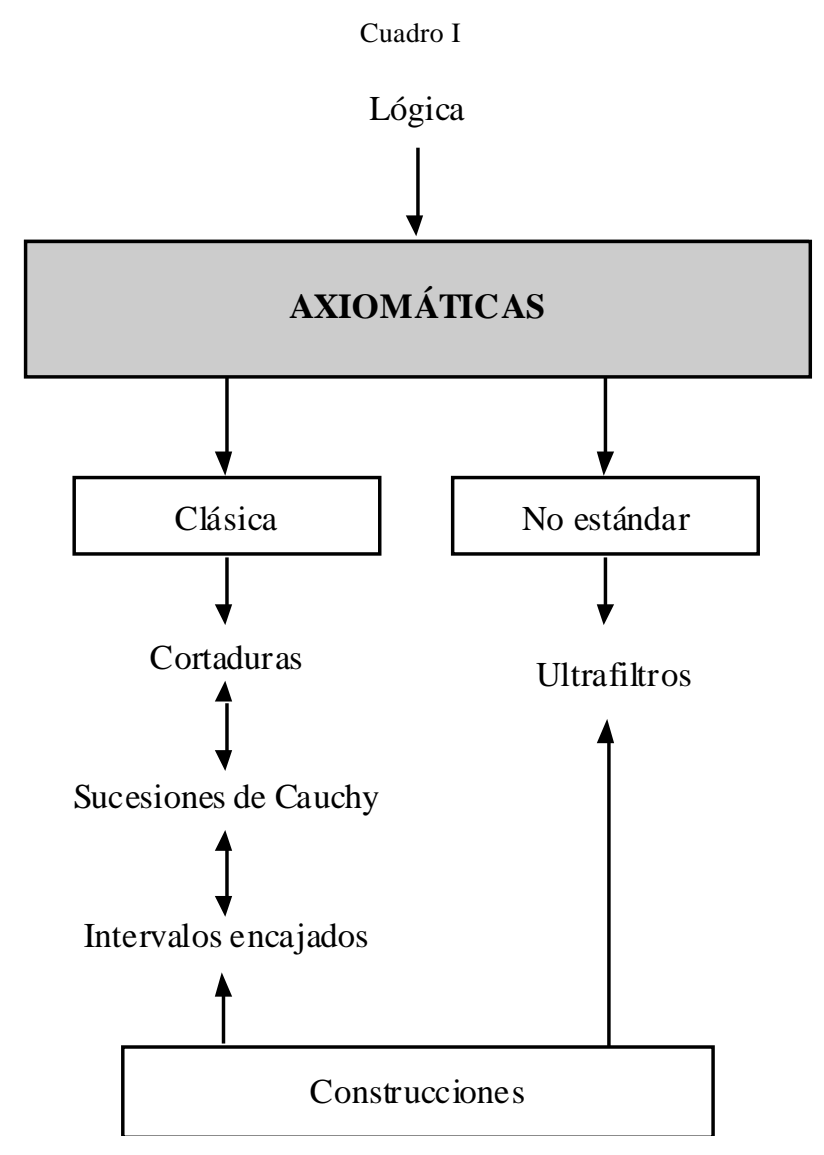

ramos con certeza que el espacio es discontinuo, sin duda nada nos podría impedir, si así lo quisiéramos, que lo hiciéramos continuo en el pensamiento rellenando sus lagunas. Pero esta compleción consistiría en una creación de individuos-punto y habría de realizarse de acuerdo con el principio anterior». (p. 85)

En el prólogo del trabajo, Dedekind expresa que encuentra en el ya citado axioma de Cantor una afirmación idéntica a la suya (aunque formulada en otros términos) respecto de lo que constituye la esencia de la continuidad.

Crossley (1987, p. 150) afirma que «no hay manera de verificar cuál es la estructura "real" (en el sentido de genuina) de la recta geométrica». Diferentes matemáticos han desarrollado estructuras numéricas que, basándose en una elección axiomática adecuada, permiten, utilizarla como modelo de esas estructuras. Solomon (1991) reconoce esta posibilidad de adecuación de la recta a diferentes estructuras cuando afirma: «En un sentido, la "verdad" acerca de la naturaleza de la recta es una cuestión referida a cómo formalizamos las propiedades de la recta tal que sean consistentes con las propiedades de la matemática.»

En el análisis no estándar de Robinson (1974) se trabaja con una estructura numérica (el sistema de números hiperreales) constituida por los números reales a los que se le incorporan los (números) infinitésimos e infinitos. Los números infinitésimos tienen sentido en el marco de una axiomática, elaborada por Robinson, «más amplia» que la axiomática de $R$ (clásico) pero compatible con ella ${ }^{3}$, como ilustra el cuadro I.

- En dicha estructura, el axioma de Arquímedes no se satisface, puesto que el producto de un infinitésimo por cualquier real estándar o por otro infinitésimo es siempre menor que cualquier fracción ordinaria positiva. La recta hiperreal contiene, además de los números reales, los infinitésimos y los infinitos. Keisler (1976) representa estos números en la recta geométrica con ayuda de dos metáforas: «un microscopio infinitesimal» y «un telescopio infinito» para sugerir, respectivamente, los infinitésimos y los infinitos.

La controversia acerca de la interpretación de la recta es tratada por este autor cuando afirma: «El sistema de números reales es una creación puramente matemática que puede o no dar una imagen precisa de una línea recta en el espacio físico. El hecho es que, mientras que nuestros sentidos nos dan una muy buena idea de cómo son los segmentos de recta de tamaño medio, sabemos muy poco acerca de cómo son, en el espacio físico, segmentos de recta muy grandes o muy pequeños. Por otro lado, hasta dónde podemos contar, la recta real es bastante como una recta en el espacio físico para todos los propósitos prácticos, y es fácil trabajar con ella. La recta real es, por lo tanto un, "modelo matemático" útil de una recta en el espacio.» (p. 1)

Casi medio siglo antes de que se concretara la formalización de los hiperreales, Veronese (1994) analizaba desde el punto de vista geométrico (no aritmético) la estructura de la línea recta, afirmando que el «axioma» de Arquímedes puede ser deducido del postulado del continuo dado por Weierstrass y Cantor. Veronese formula otro axioma del continuo (lo llama postulado) en los siguientes términos: "Si en un segmento $\mathrm{AB}$ existe un segmento variable $\mathrm{xx}$ ' tal que Ax es siempre creciente y más pequeño que Ax', que es siempre decreciente, y Xx' pasa a ser infinitamente pequeño (es decir, más pequeño que cualquier segmento dado), entonces $\mathrm{xx}$ ' contiene un punto Y distinto de X y X'.» (p. 171)

A partir de este axioma, Veronese construye segmentos actualmente infinitos e infinitesimales. Estos últimos satisfacen las propiedades fundamentales de la línea recta, exceptuando el axioma de Arquímedes. Respecto de la interpretación de la recta, Veronese afirma: «El postulado según el cual un punto corresponde a cada número racional no está verificado en la práctica y, si se idealiza el punto y el segmento de tal manera que este último siempre contenga puntos distintos de sus extremos, la correspondencia uno a uno entre los puntos de la línea recta y los números reales ordinarios no está ya justificada.» (p. 171)

El análisis no estándar de Robinson y el continuo geométrico de Veronese son ejemplos de modelos matemáticos no arquimedianos que suponen interpretaciones diferentes de la interpretación estándar de la recta. 
Solomon (1991) analiza desde el punto de vista psicológico la doble naturaleza que tiene una recta para la mente humana. Hace una distinción entre la recta como «idea», que tiene propiedades contradictorias (por ejemplo, un segmento de recta puede pensarse como un conjunto de puntos, o también como un conjunto de infinitesimales) y la recta como «objeto físico» (un segmento de recta en el espacio físico es un conjunto finito de puntos discretos). «Discreto y continuo son propiedades que precipitan cuando adoptamos un punto de vista, al examinar la recta». Este autor concluye que la naturaleza dual de la recta puede tomarse como un símbolo de la naturaleza dual de la mente. «Hay niveles de significado y de experiencia más profundos que están disponibles si podemos aprender a pensar más profundamente, y también a poner el pensamiento a un lado -ir más allá de la lógica.»

Desde el punto de vista matemático, las interpretaciones de la recta se justifican mediante distintas formulaciones axiomáticas. Como se pone de manifiesto en las afirmaciones anteriores, sus respectivos defensores son conscientes de que no están descubriendo una «naturaleza verdadera de la recta»; sólo realizan una elección axiomática que permite justificar (siguiendo una serie de inferencias válidas) los resultados posteriores.

Pensamos que las intuiciones son el soporte de estas elecciones axiomáticas ${ }^{4,5}$. Los textos que hemos recogido ponen de manifiesto que hay axiomáticas bien compatibles o bien incompatibles con determinadas intuiciones y que una discusión inicial de carácter intuitivo sirve de soporte a posteriores elaboraciones axiomáticas. (En la mayoría de los casos, dichas intuiciones quedan ocultas por el deslizamiento semántico producido al usar los mismos términos.)

A título ilustrativo, consideraremos exclusivamente una pregunta: La recta, ¿se compone de puntos o no? La respuesta es resultado de una intuición.

La respuesta negativa tiene su origen en Aristóteles (1995, p. 336): «[...] es imposible que algo continuo esté hecho de indivisibles, como, por ejemplo, que una línea esté hecha de puntos, si damos por supuesto que la línea es un continuo y el punto un indivisible». También Veronese acepta una respuesta negativa: «Cuando extraemos una parte de un continuo, introducimos signos o «puntos» para marcar los extremos de las partes en las que descomponemos el continuo. Consideramos que los puntos no tienen partes. No necesitamos considerar que los puntos son por sí mismos partes del continuo, sino sólo entidades mentales auxiliares que indican dónde se unen las partes del continuo. El propio continuo no se compone de puntos». (Citado en Fisher, 1994, p. 115)

La respuesta afirmativa tiene su origen más claro en la aportación de Dedekind, como se desprende de las citas textuales dadas más arriba.

En ambos casos, aunque los enunciados intuitivos no sirven para hacer matemáticas, guían a los autores en su búsqueda de afirmaciones axiomáticas.

\section{CUESTIONES FENOMENOLÓGICAS. UN ANÁLISIS CONCEPTUAL}

Para establecer una biyección entre puntos de la recta y números reales, hemos de aceptar dos supuestos: a) La recta se compone de puntos. $b$ ) La linealidad geométrica se describe mediante la estructura de espacio vectorial de dimensión 1 sobre el cuerpo $R$.

Tradicionalmente, la ley que rige la correspondencia números reales - puntos de la recta se apoya en la medida de longitudes: fijados dos puntos cualesquiera, $O$ e $I$, designados con los números 0 y 1 , respectivamente, a todo número real $r$ le corresponde un único punto $M$ de la recta, tal que $O M=r O I$. El vector $O M$ es igual al producto del real $r$ por el vector $O I ; r$ se llama abscisa del punto $M$ y $|r|$ corresponde a la medida del segmento $O M$ según la unidad $O I$.

Fowler (1992) establece la biyección números reales puntos de la recta mediante un sistema de etiquetado. A partir de dos puntos cualesquiera, etiquetados con 0 y 1 respectivamente y mediante el algoritmo de Euclides ${ }^{6}$, «el conjunto de números reales será el conjunto de todas las posibles etiquetas, tal que las etiquetas determinarán lo que concebimos como los puntos de la recta y las propiedades de estas etiquetas determinarán las propiedades geométricas de la recta».

En general, se acepta que la recta geométrica exhibe el orden continuo y total de $R^{7}$.

El estudio que sigue se refiere a la primera biyección mencionada, porque usualmente es la que se utiliza en el sistema escolar. El término etiqueta, por tanto, no remite al enfoque de Fowler, sino al emparejamiento de puntos y números a través de alguna medición.

Una estrategia sencilla consiste en analizar cómo, dado un punto de la recta, se llega a «su» abscisa y cómo, dado un número real, se llega a su «marca» puntual. Sin embargo, previamente conviene analizar las expresiones punto dado y número dado.

El sentido de dar un punto (para determinar el número real asociado) exige dos «operaciones» complementarias: la de distinguir en la recta ese punto con alguna marca y suponer dadas otras dos marcas correspondientes al origen y unidad; en este caso, el problema de medida consiste en determinar $r$, conocidos $O, I$ y $M$.

Si el dato es el número, el problema de medida consiste en determinar $M$ conocidos $O$, $I$ y $r$. Ahora bien, para «dar un número» necesitamos una descripción inequívoca de $r$ (como en $« r=\operatorname{dos} » 0$ o $\langle r=0$ '333...»). Esa descripción se apoya al menos en una representación (por ejemplo: verbal, base diez, fracción continua, icónica). Ninguna representación permite describir inequívocamente todos los números reales; por ejemplo, el conjunto de números decimales de hasta $n$ cifras, $D_{n}$, es numerable, mientras que $R$ no lo es. Sabemos describir acaso todos los algebraicos así como muchos números trascendentes. (Algunos computables son trascenden- 
tes, como 0'123456789101112... ${ }^{8}$ Algunos trascendentes no son computables.)

Por consiguiente, la tarea que vamos a abordar no puede realizarse exhaustivamente (ni para todo número real, porque hay muchos números reales que no sabemos describir inequívocamente, ni para todo punto, porque no sabríamos la descripción de su abscisa).

También conviene observar que, supuesta una descripción inequívoca de un número real, no tenemos garantía de expresarla en términos de medidas. Así, la fracción continua infinita $[1 ; 1,1,1 \ldots]$ carece de significado métrico; sólo lo adquiere cuando establecemos que se trata de una representación del número áureo.

\section{Determinación del punto correspondiente a un nú- mero dado}

Fijados los puntos correspondientes a 0 y 1 , el siguiente paso en la representación de un número real cualquiera es determinar el correspondiente punto de la recta. Tratándose de un procedimiento de medición, la manipulación con instrumentos físicos (como la regla graduada, el micrómetro, la regla de un solo borde y el compás o el intégrafo) para determinar la posición de un número real sobre una recta produce siempre un resultado aproximado. Pasamos, por tanto, revista a dos instrumentos ideales: la regla y el compás, y el intégrafo.

- Es posible representar exactamente números reales mediante procedimientos geométricos que involucran el uso de la regla y el compás ideales. Estos números están bien estudiados; se denominan constructibles y constituyen un subcuerpo de $R$ estable por la raíz cuadrada ${ }^{10}$ (Carrega, 1981).

- Para determinar puntos asociados a números reales con un intégrafo (Puig Adam, 1962, pp. 109-110), se necesita un buen conocimiento de la integral de Riemann y de algunos movimientos en el plano (giros y traslaciones) que posibilitan la obtención progresiva de una primitiva a medida que se recorre la gráfica de una función. Cabe imaginar simulaciones (no ideales) de este artefacto basadas en medios informáticos. Si suponemos que la gráfica de la función continua dada constituye una representación exacta de dicha función, entonces un intégrafo ideal permitiría construir segmentos de longitud arbitraria $^{11}$.

En resumen: hay métodos para determinar el punto de la recta correspondiente a un real dado y dependen de la representación de éste. Todos los números constructibles con regla y compás admiten una representación idealmente exacta; los restantes números (sean algebraicos o trascendentes) no admiten hoy día una representación idealmente exacta. El uso del intégrafo para «cualquier» número o la definición de números algebraicos mediante procedimientos finitos (ver Recio, 1998; pp. 101-150) no tienen sentido sin un buen conocimiento previo del número real.

\section{Determinación del número real correspondiente a un punto dado}

La identificación del número real que corresponde a un punto dado $M$, fijados el origen y la unidad de medida, desde el punto de vista físico es siempre aproximada.

- Cuando se conoce alguna relación entre los puntos que corresponden al origen, la unidad y el punto $M$, podemos hablar de una medida indirecta, pues está basada en una relación que comúnmente es geométrica. En este caso, desde el punto de vista «ideal», la determinación del número real es exacta, aunque en la práctica es posible que se produzca algún error y la medición resulte, en el plano físico, aproximada. Veamos un ejemplo de medición indirecta. En la figura 1, se ha construido una recta y se han marcado tres puntos sobre ella, $O, I$ y $M$, de modo que la distancia entre $I$ y $M$ es el doble de la distancia entre $O$ e $I$. La información proporcionada es suficiente para determinar la abscisa de $M, 3$, pero la relación dada entre las longitudes de los segmentos $O I$ e $I M$ juega un papel crítico.

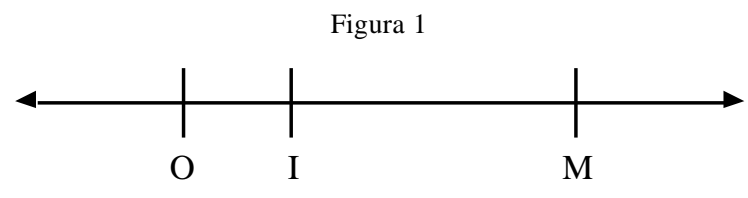

- Cuando no se conoce relación alguna entre los puntos correspondientes al origen, la unidad y el punto $M$, la medición es aproximada, y se debe recurrir a instrumentos de medida. En este caso la medición es directa, por aplicación sucesiva del segmento unidad. Se trata de identificar, en principio, el intervalo de extremos enteros al que pertenece el punto $M$. Si este punto coincide con algún extremo del intervalo, el trabajo ha terminado. Si no es así, es posible realizar subdivisiones sucesivas en el intervalo, para mejorar cuanto sea posible la estimación del real correspondiente a $M$. La figura 2 presenta un ejemplo de este tipo de medición; conocemos los puntos $O$ e $I$, correspondientes a 0 y 1 .

Figura 2

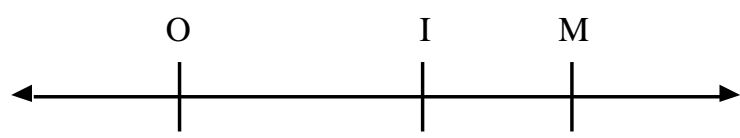

A simple vista podemos afirmar que la abscisa de $M$ está comprendida entre 1 y 2 . Si afinamos la observación y recurrimos a algún instrumento de medición (como una regla graduada o un compás), podemos mejorar nuestra estimación numérica, pero siempre estamos limitados por la potencia del instrumento utilizado y por las características físicas del gráfico (constituido por un trazo de tinta de espesor variable sobre una hoja de papel).

Teniendo en cuenta las distancias que indica el procesador de texto empleado, entre los puntos $O$ e $I(1,80 \mathrm{~cm})$ e $I$ y $M(1,20 \mathrm{~cm})$ de la figura 2 , hemos conseguido 
etiquetar $M$ con la abscisa $5 / 3$. Sin embargo, esas distancias están calculadas con una aproximación de dos cifras decimales. Si dispusiésemos de un programa más preciso, la identificación numérica del punto $M$ (figura 2) sería algo diferente (la diferencia en valor absoluto dependerá de la aproximación decimal que ese nuevo programa permita).

Las medidas directas se agotan habitualmente en $D_{2} \mathrm{o}$ $D_{3}$. Es posible proseguir el proceso de subdividir la unidad, pero no es seguro que se admita la posibilidad de un proceso infinito. Romero (1995), en su descripción de las actividades de medición y representación en la recta de números irracionales, llevada a cabo con alumnos de 14-15 años, expresa: «Cuando yo intento diferenciar entre el plano físico, en el que están llevando a cabo la conmensuración, y el plano ideal, en el que dicha conmensuración se prolongaría infinitamente, ya que no existiría una parte alícuota que permitiera dar un resultado en forma de fracción, de forma que se puedan integrar ambos modos de medida, los alumnos quedan fuera de este tipo de discurso.» (p. 231)

En educación es preciso suscitar la posibilidad de un proceso infinito, que justifique el abandono del marco de la medida directa, y considerar relaciones geométricas entre cantidades, es decir, recurrir a la medición indirecta. A título de ejemplo, consideremos la representación en la recta del número 1/11. La división de 1 entre 11 conduce a un proceso infinito, caracterizado por la repetición del patrón «resto parcial igual a $1 »$, y conduce al cociente exacto 0'09090909... La aceptación de este proceso infinito es clave en el paso de la representación fraccionaria a la decimal. Las diferentes cifras del cociente se pueden traducir a operaciones de medida: desde la marca 0 , marcar 9 centésimas; desde esta marca, marcar nueve diezmilésimas, y así, sucesivamente. Sin embargo, este proceso infinito de medida no permite obtener una representación exacta en la recta (en la práctica, las marcas acabarían por «superponerse»; en la teoría, ignoramos cómo este proceso de medición directa podría conducirnos hasta el límite). Para conseguir esa representación exacta tenemos que recurrir a relaciones geométricas elementales (en este caso, el teorema de Tales) que permiten dividir un segmento unidad en once partes iguales. (No se debe olvidar que hay infinitos números reales (uno de ellos se describe en la nota 8) para cuya representación en la recta no disponemos de relaciones geométricas.)

Como resultado de este estudio, concluimos que el establecimiento de una relación métrica entre dos de los segmentos determinados por tres puntos es una condición necesaria y suficiente para determinar de modo exacto el número real correspondiente a uno de esos tres puntos. No hemos encontrado ninguna otra posibilidad. En esto se apoya, por ejemplo, el físico, para escribir fórmulas con cantidades que en la práctica no se pueden medir con total precisión ${ }^{12}$.

La representación en la recta de los números reales está ligada a la aceptación intuitiva de que las marcas sobre la recta corresponden a los números indicados, pero la coincidencia entre las marcas geométricas y las etiquetas numéricas difícilmente será «exacta».

Como las mediciones reales son siempre aproximadas, sólo es posible hablar de una medición exacta desde un punto de vista ideal, cuando se realizan mediciones indirectas, conociendo una relación geométrica, o mediante construcciones con instrumentos geométricos (regla y compás ideales $)^{13}$.

\section{Conclusiones}

Resumiendo las ideas anteriores:

1) La identificación punto-número «para cualquier número real» es esencialmente aproximada o exige un buen conocimiento de $R$.

2) «Físicamente», la identificación punto-número nunca es exacta.

3) Para que la representación sea «idealmente» exacta, es necesario que se dé una condición suplementaria: o bien el número dado es constructible con regla y compás; o bien el tercer punto dado se relaciona con los correspondientes a 0 y 1 mediante una relación explícita, que es equivalente a la constructibilidad con regla y compás.

Como consecuencia, pensamos que, en educación secundaria, hay razones para afirmar lo siguiente:

1) La asignación concreta punto-número se apoya en dos supuestos: a) la recta está formada por puntos; b) a cada número real le corresponde un único punto y recíprocamente. En educación secundaria, el segundo supuesto se genera «intuyendo» o «decretando» que la constructibilidad se generaliza a todo número real.

2) No hay inconveniente en admitir que puedan inventarse procedimientos finitos para asociar exactamente puntos de la recta y números reales no constructibles con regla y compás. En este caso, es necesario ampliar la noción de punto-número constructible, pero de manera que esta ampliación no implique un buen conocimiento previo de $R$ (el cual, precisamente, se está estudiando).

\section{REPRESENTACIÓN EN LA RECTA Y OTRAS REPRESENTACIONES}

En este apartado repasamos brevemente algunas diferencias entre las representaciones simbólicas y gráficas de los números reales y la representación de éstos en la recta, con objeto de justificar la conjetura enunciada en la introducción. En el caso de las representaciones gráficas, hemos limitado el estudio comparativo a la relación entre parte y todo porque ésta es la única que admite enfoques continuos (cosa que no ocurre con otras representaciones gráficas, como los números figurados). Hemos incluido una colección de características que consideramos distintivas de la representación en la recta. 


\section{Representación en la recta y representaciones simbó- licas}

1) La notación decimal constituye una herramienta esencial para representar números a pesar de que no existe biyección entre $D_{n}$ y $R$.

2) Las representaciones simbólicas no siempre permiten visualizar la noción de orden. La representación simbólica de los números reales mediante la notación decimal, por ejemplo, permite ordenar dos números dados, aunque en algunos casos no se logre «de un vistazo» el reconocimiento de qué número es mayor, como ocurre con la pareja 0’4899899 y 0’4898999.

La comparación de dos fracciones se realiza recurriendo a un algoritmo. La comparación de dos radicales de igual índice se realiza directamente comparando los respectivos radicandos. Cuando se comparan números reales expresados con diferentes notaciones simbólicas, el trabajo es más complejo y es necesario, muchas veces, usar una representación común, como ocurre en los siguientes casos: $\sqrt{2}$ y $17 / 12 ; \pi$ y $22 / 7$.

3) Para indicar el número real que corresponde a un punto de la recta, es necesario recurrir a una representación simbólica, como, por ejemplo, la notación fraccionaria, la notación en el sistema decimal o la notación operatoria habitual de radicales. Sin estas representaciones simbólicas, la marca en la recta sería imposible de interpretar.

La notación fraccionaria $(2 / 3)$ y la de radical $(\sqrt{ } 2)$ se apoyan, a su vez, en la notación decimal. Numerador, denominador, índice y radicando están formados por números expresados en el sistema decimal $(2,3,2,2$, respectivamente). En estos casos, un algoritmo u operación permite pasar a una representación decimal aproximada de dichos números. Sin embargo, la diferencia que se establece con la representación en la recta es que cualquiera de estas combinaciones de símbolos $(2 / 3, \sqrt{2})$ representa un único número que queda identificado completamente con esa representación. Un punto sobre la recta debe asociarse a alguna etiqueta simbólica para que se produzca la representación del número real así simbolizado en aquélla ${ }^{14}$.

4) Un número admite representaciones equivalentes $\left(2 / 3=4 / 6 ; 2^{1 / 2}=2^{2 / 4} \ldots\right)$, mientras que los puntos son idénticos e indiferenciados. Aunque cualquier representación simbólica inequívoca identifique un único número real, en la recta se necesita una pareja (punto resaltado -representación simbólica) para identificar la asociación única punto-número.

\section{Representación en la recta y relación entre parte y todo}

Los gráficos utilizados para expresar la relación entre parte y todo sólo comparten con la representación en la recta la idea de asociar un número a un signo o gráfico (un punto sobre la recta o una figura geométrica dividida en su interior). Las diferencias entre ambos modos de representación gráfica son diversas. He aquí las más destacables:

1) Cada gráfico de la relación entre parte y todo representa unos pocos números reales (constructibles o no). La recta, en cambio, es un modelo del conjunto de números reales.

Figura 3
A

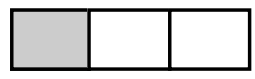

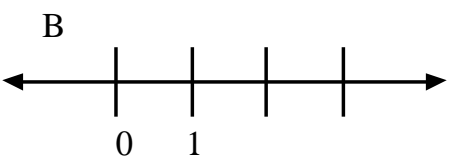

La figura $3 \mathrm{~A}$ representa la relación constructible entre tres números reales: $x / 3,2 x / 3$ y $x$. En $3 \mathrm{~B}$ sólo hay etiquetados dos puntos y resaltados otros dos, que identificamos fácilmente con los números 2 y 3 . No obstante, al observar ese segmento, imaginamos que contiene todos los números reales.

2) En los gráficos que expresan la relación entre parte y todo, no siempre es necesario acudir a un sistema de representación simbólico para reconocer al número que se está representando. El gráfico de $3 \mathrm{~A}$ posee la información necesaria para identificar tres números (normalmente, $1,1 / 3$ y $2 / 3$ ). En la representación en la recta numérica, la identificación de los puntos necesita de algún tipo de representación simbólica (con excepción, acaso, de algunos enteros).

3) Una representación como la de 3A permite comparar un todo con algunas de sus partes $(\pi>\pi / 3, \pi>2 \pi / 3)$ o algunas partes de un todo $(2 \pi / 3>\pi / 3)$. Sin embargo, no exhibe necesariamente el orden de las partes.

\section{Algunas características de la representación en la recta}

Resumimos las características de la representación en la recta que, según se desprende de lo dicho, consideramos esenciales en apoyo de la conjetura enunciada en la introducción.

1) Aceptando el axioma de Cantor, la recta se identifica con el conjunto ordenado de los números reales (Crossley, 1987). Permite, en principio, representarlos todos, uno por uno, mediante puntos.

2) La representación en la recta ayuda a intuir el orden continuo y total de $R$.

3) La representación de un número en la recta se apoya en un procedimiento de medida de longitudes mediante el cual es posible resaltar un punto y atribuirle una representación simbólica correspondiente a algún sistema de representación de números. Hemos presentado ejemplos en que la atribución numérica de los puntos resaltados fue posible gracias al uso de uno de los 
sistemas de representación (decimal, fraccionaria o icónica).

4) La ausencia de exactitud en las representaciones de reales en la recta, realizadas con diferentes instrumentos, supone reconocer la diferencia entre abscisa y etiqueta asignada. Difícilmente la etiqueta correspondiente a un punto «marcado» sobre la recta coincidirá con la abscisa correspondiente a dicho punto (Notas 8 y 9), con excepción de las etiquetas correspondientes a 0 y 1 , que son fijadas de antemano. Bachelard (1969, p. 211) denomina obstáculos del conocimiento cuantitativo a la posibilidad de obtener resultados erróneos como consecuencia de un conocimiento inmediato que es subjetivo. Un conocimiento adecuado de la representación en la recta exige la adquisición de criterios de «tolerancia» para efectuar la mencionada distinción.

5) Cada uno de los segmentos de recta sobre los que se representan unos pocos números (el origen, la unidad y algunos otros) está simbolizando el conjunto de números reales en su totalidad. La representación en la recta permite «actualizar» en un segmento (como en la figura 3B la totalidad del conjunto de números reales.

6) La continuidad intuitiva de la recta permite expresar la continuidad de $R$ (la cual se manifiesta por el axioma de completitud o proposición equivalente) pero también permite expresar la continuidad de otros conjuntos «más amplios» (como el de los hiperreales).

Aceptando, como hipótesis de trabajo, la conjetura de que la representación en la recta es más compleja (conceptual y procedimentalmente) que otras representaciones de los números reales, y explicitado el sentido de la diferencia entre dichas representaciones, se deducen algunas de las precauciones con que la representación en la recta debe trabajarse en educación secundaria. Estas precauciones atañen a la «naturaleza» de la recta y a las intuiciones que soportan una concepción de la recta geométrica, a las dificultades conceptuales y procedimentales de la asignación punto-número y a la imposibilidad de realizar esta asignación en su totalidad (salvo como creencia soportada por una generalización).

\section{AGRADECIMIENTOS}

Ma. José González López nos convenció de que la expresión número dado no podía admitirse sin un análisis, algunos de cuyos elementos actuales comentó para nosotros. Luis Rico Romero ayudó a desentrañar frases confusas y a liberar ideas de sus sobreentendidos. Ambos son «culpables» de tomarse nuestro trabajo con interés y aprecio, pero no de los errores o confusiones aún presentes.

\begin{abstract}
NOTAS
${ }^{1}$ La siguiente cita (adaptada, con ayuda de corchetes) de Truss (1997,p. 112) expone claramente la cuestión: «La construcción de los números reales a partir de $Q$ [plantea] algunos problemas conceptuales que no se dan en $Z$ y $Q$. La no-numerabilidad (uncountability) de $R$ se discutirá en la sección siguiente. Aquí queremos enfocar otro punto, y es que el nuevo axioma que estamos exigiendo que cumpla $R$ es «de segundo orden». [En el primer orden,] las variables deberían exclusivamente recorrer los elementos del dominio que se está considerando. Éste no es el caso [con los números reales]. La afirmación de que $R$ es completamente ordenado(order-complete) implicacuantificadores sobre subconjuntos de $R$ : «[...] para todo subconjunto $X$ de $R$, si $X$ es no vacío y acotado superiormente, entonces [...]». Este es un axioma de segundo orden. Más precisamente, una fórmula es de segundo orden si todas las variables recorren el dominio o sus subconjuntos; $[\ldots] \gg$.
\end{abstract}

Se comprende que, en bachillerato, resulte difícil o imposible trabajar cabalmente el axioma de completitud.
${ }^{2}$ Esta selección elude dos destacadas aportaciones: 1) Como aceptamos el infinito actual, no hemos incluido las matemáticas llamadas constructivas (Bishop y Bridges, 1985) ni las poderosas intuiciones elaboradas sobre el «continuo constructivista». Por ejemplo, Van Dalen (1997) describe el continuo intuicionista como «indescomponible» y demuestra que, «si se quita un punto del continuo intuicionista, quedan puntos de los que no podemos saber si pertenecen o no a la parte restante». 2) Hemos descartado también la mención de los números surreales de Conway. Aunque se han publicado presentaciones de estos números en forma de cuentos (Knuth, 1979) y el acercamiento puede hacerse a través de la teoría de juegos (Mainzer, 1990), las demandas algebraicas de su construcción parecen excesivamente complejas para usarlas fuera de la universidad y, sobre todo, antes de conocer los números reales.

${ }^{3}$ En el capítulo histórico de su libro, Robinson relaciona sus infinitésimos con las cantidades infinitesimales y evanescentes que estuvieron tan al uso a lo largo de los siglos XVII y XVIII, pero 
hoy por hoy no estamos en condiciones de «ver» los infinitésimos (Kossak, 1996).

${ }^{4}$ Al usar el término intuición entramos en un campo psicológico inevitablemente complejo, en el que no somos expertos. Hasta donde somos capaces de controlar el sentido de este término, seguimos a Fischbein (1987).

${ }^{5}$ Esto no equivale a decir que «cada axioma» enuncie formalmente una intuición ni que cada conjunto de axiomas corresponda a un «conjunto» dado de intuiciones. El lenguaje matemático de hoy no tolera enunciados que puedan calificarse de «psicológicamente vagos»; el matemático que quiere discutir o ampliar una axiomática no tiene mas remedio que enunciar otra axiomática y extraer consecuencias lógicas de ella.

${ }^{6}$ Considerando un punto $X_{0}$ de una semirrecta, se escribe $x_{0}$ para todo segmento congruente a $O X_{0}, x$ para 01 . Se expresa: $x_{0}=n_{0} x_{1}+x_{2} \operatorname{con} x_{2}<x_{1}, x_{1}=n_{1} X_{2}+X_{3} \operatorname{con} X_{3}<X_{2}$, etc. $\mathrm{Si}$ en algún estado no hay resto, el proceso termina. Así, se descomponen $0 X_{0}$ y 01 en subintervalos y se pueden usar $\operatorname{los} n$ para etiquetar el punto $X_{0}$. Se escribe $X_{0}=\left[n_{0}, n_{1}, n_{2} \ldots\right]$ (Fowler, 1992 , p. 726). El proceso no termina cuando no hay unidad común de medida entre el segmento unidad y el segmento considerado, como se explica en Gardiner (1982, pp. 53-54). El etiquetado así definido no origina el etiquetado habitual, consistente en la repetición de una unidad y, cuando es necesario, una subdivisión de la unidad. En el procedimiento propuesto por Fowler, las etiquetas de cada punto se corresponden con los sucesivos divisores obtenidos en el algoritmo de Euclides.

${ }^{7}$ Describimos cómodamente (pero negativamente) el orden continuo de $R$ (y de la recta de puntos) diciendo que no hay ningún método que permita asignar un sucesor a todo número real (ni a todo punto). Por lo que respecta al orden total, elegida la orientación habitual, decir que un punto $A$ está a la izquierda de otro punto $B$ equivale a decir de sus respectivas abscisas ( $r$ y $r$ ') que $r<r$ '. Esto permite asociar ordenadamente cada número real con «su» correspondiente punto de la recta en la que previamente se han marcado el origen y la unidad: $\operatorname{si} r<r$ ', el punto asociado $a r+a$ está a la izquierda de $r$ ' $+a$, cualesquiera que sean los números $r, r^{\prime}, a$. Si, además, $a>0$, también $r a$ está a la izquierda de $r^{\prime} a$.

${ }^{8}$ Esta escritura decimal es equívoca; se apoya en un procedimiento implícito que el lector habrá seguramente adivinado: concatenar, desde la coma decimal, todos los números naturales en base diez. En este caso, el número es computable porque el procedimiento permite predecir, para todo $n$, la cifra $n$-ésima del número.
${ }^{9}$ Para disponer de un dado en base diez podemos usar un icosaedro y marcar dos caras con el 0 , otras dos con el 1 , etc. Decidamos «construir un número real» del siguiente modo. Su parte entera será 0 y su cifra decimal de orden $10^{-n}$ será la que indique el $n$-ésimo lanzamiento del dado. Este número no es computable, ya que no se conoce ningún algoritmo que permita predecir su cifra $n$-ésima.

${ }^{10}$ A este subcuerpo de $R$ pertenecen, por ejemplo, todos los racionales y las raíces de índice $2^{n}$ de los racionales positivos, pero no pertenecen, por ejemplo, los radicales (simplificados) de índice impar, como $2^{1 / 3}$. Así, con regla y compás, no es posible construir exactamente un cubo de volumen $2 \mathrm{~m}^{3}$.

${ }^{11}$ Pueden consultarse indicaciones para construir un segmento de longitud $s$ en Coriat y otros (1989, pp. 135-136).

${ }^{12}$ Por ejemplo, la fórmula del período de un péndulo simple, $T=2 \pi \sqrt{ }(I / g)$, donde $I$ representa la longitud del péndulo y g la aceleración de la gravedad, informa de que hay una relación exacta (en la recta geométrica interpretada como eje temporal) entre el origen, la unidad y el período. No importa que los valores de $I$ y $g$ se obtengan como resultado de una medida o se declaren «pordefinición» exactamente iguales, respectivamente, por ejemplo, a $1 \mathrm{~m}$ y 9' $81 \mathrm{~m} / \mathrm{s}^{2}$, ni que se piense en $\pi$ como igual a 3'14 o a la fracción exacta entre la longitud de una circunferencia y su diámetro: en la práctica, la medida de $T$ será siempre aproximada y constará de unas pocas cifras decimales.

${ }^{13}$ Para controlar la precisión de una medida se utilizan las nociones de error absoluto y error relativo. Dado un número real $x$, que representa la «medida exacta» y siendo $w$ el valor obtenido en una medición, el error absoluto se define como $|x-w|$. El error relativo es igual al cociente entre el error absoluto y el casi siempre inaccesible valor exacto, es decir, $|x-w| / x$.

${ }^{14}$ Como ya se indicó, precisamente $2 / 3$ y $\sqrt{ } 2$ admiten una marca idealmente exacta. No ocurre lo mismo con las expresiones decimales correspondientes 0'666666666... y 1'41421356... si no se conoce el proceso que permite identificar estas representaciones decimales con sus respectivas representaciones simbólicas equivalentes.

${ }^{15}$ Es posible establecer diferentes biyecciones de $R$ sobre ]-1,1[; por ejemplo, usando la función arco tangente. Aquí lo único que pretendemos enfatizar es la idea de totalidad suscitada por un segmento.

\section{REFERENCIAS BIBLIOGRÁFICAS}

ARISTÓTELES (1995). Física. Trad. de Echandía, G.R. de. Madrid: Gredos.

BACHELARD, G. (1969). La Formation de l'Esprit Scientifique. París: Vrin.

BELNA, J.P. (1996). La notion de nombre chez Dedekind, Cantor, Frege. Théories, conceptions et philosophie. París: Vrin.
BOUVIER, A. y GEORGE, M. (1984): Diccionario de matemáticas. Trad. de Armiño, M. y Bordoy, V. Madrid: Akal.

BISHOP, E. y BRIDGES, D. (1985). Constructive Analysis. Berlín: Springer-Verlag.

CARREGA, J.C. (1981). Théorie des corps. La règle et le compas. París: Hermann. 
CORIAT, M., GARCÍA, C., LARA, A., PÉREZ, A., PÉREZ, R., SANDOVAL, P. y VELA, M. (1989).Seis para cuadrar. Madrid: MEC.

CROSSLEY, J.N. (1987). The emergence of number. Singapur: World Scientific.

DEDEKIND, R. (1998). ¿Qué son y para qué sirven los números? $Y$ otros escritos sobre los fundamentos de la matemática. Trad. de Ferreirós, J. Madrid: Alianza Editorial.

FISCHBEIN, E. (1987). Intuition in Science and Mathematics. An Educational Approach. Dordrecht: Reidel Pub. Co.

FISHER, G. (1994). Veronese's Non-Archimedean Linear Continuum, en Ehrlich,P.(ed.).Real numbers, generalizations of the reals, and theories of continua, pp. 107-145. Dordrecht: Kluwer Academic Publishers.

FOWLER,D. (1992). Dedekind's theorem: $\sqrt{2} \cdot \sqrt{3}=\sqrt{6}$. American Mathematical Monthly, 99(8), pp. 725-733.

GARDINER, A. (1982). Infinite Processes. Background to Analysis. Nueva York: Springer-Verlag.

KEISLER, H.J. (1976). Elementary Calculus. Boston: Prindle, Weber \& Schmidt Incorporated.

KNUTH, D.E. (1979). Números surreales. Trad. de Garriga, L. Barcelona: Reverté.

KOSSAK, R. (1996). What are infinitesimals and why they cannot be seen? American Mathematical Monthly, 103(10), pp. 846-853.

MAINZER, K. (1990). Real Numbers, en Ebbinghaus et al. (eds.). Numbers, pp. 28-53. Nueva York: Springer-Verlag.

MANSFIELD, H. (1985). Points, lines, and their representations. For the Learning of Mathematics, 5, pp. 2-6.
PUIG ADAM, P. (1962). Curso teórico práctico de cálculo integral aplicado a la física y a la técnica. Madrid: Biblioteca Matemática.

RECIO, T. (1998). Cálculo simbólico y geométrico. Madrid: Síntesis.

ROBINSON, A. (1974). Non-StandardAnalysis. (Ed. revisada). Amsterdam: North-Holland Pub. Co.

ROMERO, C. (1996). Una investigación sobre los esquemas conceptuales del continuo. Ensayo de un cuestionario. Enseñanza de las Ciencias, 14(1), pp. 3-14.

ROMERO, I. (1995). La introducción del número real en educación secundaria. Tesis doctoral. Granada: Departamento de Didáctica de la Matemática. Universidad de Granada.

SCRIVEN, M. (1988). Philosophical Inquiry Methods in Education, en Jaeger, R.M. Complementary Methods for Research in Education, pp. 131-148 Washington: AERA.

SOLOMON, A. (1991). What is a line? For the Learning of Mathematics, 11(1), pp. 9-12.

TRUSS, J.K. (1997). Foundations of Mathematical Analysis. Oxford: Clarendon Press.

VAN DALEN, D. (1997). How connected is the intuitionistic continuum? The Journal of Symbolic Logic, 62(4), pp. 11471150 .

VERONESE, G. (1994). On non-Archimedean geometry, en Ehrlich, P. (ed.). Real numbers, generalizations of the reals, and theories of continua, pp. 169-187. Dordrecht: Kluwer Academic Publishers.

[Artículo recibido en marzo de 1999 y aceptado en octubre de 1999.] 